\section{Antiemetische Therapie bei Bestrahlung}

\author{
Behandlungsoptionen zur Vermeidung von Übelkeit und Erbrechen \\ im Verlauf einer Chemotherapie haben sich gut etabliert. Nun wurde \\ der Neurokinin-1-Rezeptorantagonist (NK 1 -RA) Fosaprepitant auch bei \\ Radiotherapie zur Prävention von Nausea und Emesis untersucht.
}

$\mathrm{N}$ $\mathrm{K}_{1}$-RA werden $\mathrm{u}$.a. in Kombination mit 5- $\mathrm{HT}_{3}$-RA und Dexamethason zur Prophylaxe von chemotherapiebedingter Nausea und Emesis eingesetzt. Unklar ist die Rolle dieser Medikamente dagegen noch im Rahmen einer Strahlentherapie. In einer Phase-III-Studie sollten Wirksamkeit und Sicherheit von Fosaprepitant in Kombination mit dem 5- $\mathrm{HT}_{3}$-RA Palonosetron und Dexamethason bei Patientinnen mit Zervixkarzinom untersucht werden, die über 5 Wochen eine fraktionierten Radiotherapie mit gleichzeitiger Cisplatin-Behandlung erhielten.

Im Rahmen der multinationalen, randomisierten, doppelblinden und placebokontrollierten Studie bekamen alle Teilnehmerinnen vor der wöchentlichen
Cisplatin-Gabe $\left(40 \mathrm{mg} / \mathrm{m}^{2}\right) 16 \mathrm{mg}$ Dexamethason oral sowie $0,25 \mathrm{mg}$ Palonosetron i.v. Zudem erhielten sie randomisiert vor der Cisplatin-Gabe entweder Fosaprepitant (150 mg i.v.) oder Placebo. Primärer Endpunkt der Studie war der Anteil der Patientinnen, die nach fünf Wochen anhaltend nicht unter Emesis litten. Zur Intention-to-Treat-Population gehörten alle Patientinnen, die die Studienmedikation erhielten.

Von 234 auswertbaren Patientinnen hatten 118 die Studienmedikation bekommen und 116 Placebo. Im Fosaprepitant-Arm hielt die Emesis nach 5 Wochen noch bei $48,7 \%$ der Patientinnen an, in der Placebogruppe war dies bei 65,7\% der Patientinnen der Fall. Es gab ein signifikant niedrigeres kumulatives
Risiko für Emesis in der FosaprepitantGruppe verglichen mit der Placebogruppe (Subhazard Ratio 0,58; p =0,008).

Die Behandlung wurde gut vertragen. Es gab nur wenige Nebenwirkungen vom Grad 3; die häufigste Nebenwirkung während der 5 Therapiewochen war Diarrhö (9\% unter Fosaprepitant, $5 \%$ unter Placebo). Einzige Nebenwirkung vom Grad 4 war ein Fall von Neutropenie in der Fosaprepitant-Gruppe.

Fazit: Patientinnen mit Zervixkarzinom, die eine Chemoradiotherapie erhalten, profitieren von einer Kombination aus Fosaprepitant, Palonosetron und Dexamethason. Sie leiden dann seltener unter Nausea und Emesis als Patientinnen, die lediglich Palonosetron und Dexamethason erhalten.

Christina Berndt

Ruhlmann $\mathrm{CH}$ et al. Efficacy and safety of fosaprepitant for the prevention of nausea and emesis during 5 weeks of chemoradiotherapy for cervical cancer (the GAND-emesis study): a multinational, randomised, placebo-controlled, double-blind, phase 3 trial. Lancet Oncol. 2016;17(4):509-18.

\section{Neuer Ansatz gegen Tumorkachexie}

\section{Viele Krebspatienten haben keinen Appetit und bauen deshalb körperlich ab. Das Hormon Ghrelin ist am Hungerstoffwechsel beteiligt. Ob Anamore- lin, ein Ghrelin-Rezeptoragonist, gegen Tumorkachexie helfen könnte, wurde in zwei parallel durchgeführeten Phase-III-Studien untersucht.}

B eim Hungergefühl gesunder Menschen spielt der Stoffwechsel des Hormons Ghrelin eine wichtige Rolle. Deshalb könnte der neue Ghrelin-Rezeptoragonist Anamorelin womöglich onkologischen Patienten mit Kachexie helfen. Dies wurde in zwei parallel durchgeführten randomisierten, doppelblinden und placebokontrollierten Phase-III-Studien getestet.

An der ROMANA-1- und der ROMANA-2-Studie nahmen 93 Zentren in 19 Ländern teil. Alle Patienten litten unter einem inoperablen nichtkleinzelligen Lungenkarzinom (NSCLC) im Stadium III oder IV sowie unter Kachexie, d.h. ein Patient hatte binnen sechs Monaten mindestens $5 \%$ seines Körpergewichts verloren oder sein Body-Mass-Index
(BMI) betrug weniger als $20 \mathrm{~kg} / \mathrm{m}^{2}$. Die Patienten wurden randomisiert, entweder täglich 100 mg Anamorelin oral einzunehmen oder Placebo.

Zwischen Juli 2011 und Januar 2014 wurden 484 Patienten in die ROMANA-1-Studie aufgenommen, 495 weitere Patienten zwischen Juli 2011 und Oktober 2013 in die ROMANA-2-Studie. Jeweils im Verhältnis 2:1 randomisiert erhielten sie entweder Anamorelin oder Placebo. Binnen 12 Wochen nahm die Magermasse des Körpers (Lean Body Mass, d.h. Körpermasse minus Speicherfett) bei Patienten im AnamorelinArm im Vergleich zur Kontrollgruppe signifikant zu: in der ROMANA-1-Studie im Mittel um 0,99 kg ( $\mathrm{p}<0,0001)$, in der ROMANA-2-Studie um 0,65 kg
( $\mathrm{p}<0,0001)$. Ein stärkerer Handgriff wurde jedoch nicht festgestellt, auch zeigten sich keine Unterschiede im Hinblick auf behandlungsbedingte $\mathrm{Ne}$ benwirkungen vom Grad 3/4. Die häufigste dieser Nebenwirkungen war Hyperglykämie unter Anamorelin: bei 1 von 320 Patienten in ROMANA-1 und bei 4 von 330 Patienten in ROMANA-2.

Fazit: Der Ghrelin-Rezeptoragonist Anamorelin erhöhte das Körpergewicht von Patienten mit inoperablem NSCLC in zwei Phase-III-Studien im Vergleich zu Placebo signifikant. Der Handgriff der Patienten wurde jedoch nicht fester. $\mathrm{Da}$ es bisher kaum effektive Behandlungsmöglichkeiten für Kachexie gibt, könnte Anamorelin trotz dieses überschaubaren Erfolgs eine Option für Patienten mit krebsbedingter Anorexie oder Kachexie sein.

Christina Berndt

Temel JS et al. Anamorelin in patients with nonsmall-cell lung cancer and cachexia (ROMANA 1 and ROMANA 2): results from two randomised, double-blind, phase 3 trials. Lancet Oncol. 2016;17(4):519-31. 